\title{
Norois
}

Environnement, aménagement, société

$222 \mid 2012$

Xynthia

\section{"Vimers de mer » et sociétés littorales entre Loire et Gironde (XIVe-XVIe siècle)}

"Vimers de mer" and coastal societies between the Loire and Gironde (14th-16th century)

Jean-Luc Sarrazin

\section{OpenEdition}

Journals

Édition électronique

URL : http://journals.openedition.org/norois/4034

DOI : $10.4000 /$ norois. 4034

ISBN : 978-2-7535-1843-8

ISSN : $1760-8546$

Éditeur

Presses universitaires de Rennes

Édition imprimée

Date de publication : 28 février 2012

Pagination : 91-102

ISBN : 978-2-7535-1815-5

ISSN : 0029-182X

\section{Référence électronique}

Jean-Luc Sarrazin, « « Vimers de mer » et sociétés littorales entre Loire et Gironde (XIVe-XVle siècle) », Norois [En ligne], 222 | 2012, mis en ligne le 30 mars 2014, consulté le 31 mars 2021. URL : http:// journals.openedition.org/norois/4034 ; DOI : https://doi.org/10.4000/norois.4034

(c) Tous droits réservés 
de leurs maisons, leurs utencilles, tables, bans, litz et autres choses pour y résister; autrement elle submergeroit tout le pays ${ }^{1}$.»

C'est en ces termes que les délégués de la ville de Poitiers présentent, vers 1451, la côte poitevine, aunisienne et saintongeaise du royaume de France dans un mémoire adressé au roi Charles VII pour le dissuader d'établir la gabelle en des provinces jusqu'alors soumises à un impôt moins lourd, le quart du sel. Par-delà son caractère biaisé, le passage met l'accent sur un phénomène majeur, la menace récurrente de submersion marine à laquelle sont exposées les populations des zones basses du littoral atlantique. Il éclaire également les représentations mentales, la perception, le vécu des habitants qui considèrent l'océan comme un danger potentiel et permanent.

À partir de ce double constat, le propos de l'article est de présenter, en contrepoint des études relatives au temps présent et au traumatisme provoqué par la tempête Xynthia, les submersions que l'on peut tant bien que mal repérer sur le littoral atlantique, leur impact et les réactions qu'elles ont suscitées dans les sociétés maritaines (expression de la chancellerie royale) en un passé à la fois proche et lointain. Le cadre chronologique retenu, entre le XIV ${ }^{\mathrm{e}}$ et le $\mathrm{xVI}^{\mathrm{e}}$ siècle, est précisément le moment où les « vimers de mer » selon la terminologie même des populations sortent graduellement de la pénombre documentaire. Auparavant, à la différence des pays riverains de la mer du Nord, au reste les plus touchés de tout l'hémisphère nord par ce type de phénomène (Lamb, 1991), aucune mention de tempête ou d'inondation marine ne figure dans les sources disponibles, excepté la trace mémorielle d'un tsunami ayant frappé l'île de Bouin. Quant au tournant des $\mathrm{XVI}^{\mathrm{e}}$ et $\mathrm{XVII}{ }^{\mathrm{e}}$ siècles, il marque, à l'évidence, un infléchissement dans le mode d'appropriation des zones humides à la suite de l'édit que délivre Henri IV à Humphrey Bradley en 1599. Cette flexure n'est pas sans influence sur les types de protection face à la mer. Étant donné le thème de ce présent colloque, l'espace envisagé recouvre pour l'essentiel les secteurs littoraux touchés lors de l'épisode Xynthia, de la Loire jusqu'à l'entrée de la Gironde.

1. Mémoires présentés au roi Charles VII par les délégués de la ville de Poitiers pour le détourner d'établir la gabelle en Poitou, publiés par Ledain B. dans Archives Historiques du Poitou, t. II, Poitiers, 1873, p. 255-284.

\section{RECENSER LES VIMERS DE MER : POUR UNE BANQUE DE DONNÉES}

En moyen français des provinces de l'Ouest, le terme «vimer » ou « vimaire » (vis major, force majeure) désigne un très fort dérangement. À la fin du Moyen Âge et aux Temps Modernes, les populations parlent de « vimers de guerre » à propos des flambées d'insécurité qu'elles subissent, de «vimers de sable » ou «volemens » de sable pour évoquer les migrations de dunes, et de "vimers de mer » pour qualifier les tempêtes avec ou sans submersion marine.

Tenter de recenser les graves tempêtes ayant affecté tel ou tel secteur de la côte atlantique est évidemment la démarche première avant même d'aborder les réactions des sociétés. Pour évidente qu'elle apparaisse, l'approche se heurte à de sérieuses difficultés. Le principal obstacle est d'ordre documentaire. Un sort adverse n'a en effet cessé de peser sur la conservation des sources anciennes relatives à la côte atlantique. Pendant les guerres de religion d'une particulière intensité dans toute la région de La Rochelle, ont disparu des fonds de toute première importance. La destruction totale des archives de la vieille abbaye bénédictine de Saint-Michelen-l'Herm est ainsi une perte irrémédiable qui nous prive d'une couverture documentaire pour une zone particulièrement sensible, la baie de L'Aiguillon ainsi que les îles d'Ars et Loix en l'île de Ré qui relevaient de la seigneurie monastique. Dans l'incendie de la Chambre des comptes à Paris en 1737, ont brûlé les archives de La Rochelle et des régions circonvoisines. Des documents du plus haut intérêt comme les procès-verbaux de visite des bots dressés face à la mer n'existent plus et l'on peut supposer sans grand risque d'erreur que les fonds municipaux rochelais contenaient des renseignements sur certaines tempêtes. Les annales de La Rochelle tant celles attribuées à Nicolas Baudouin ${ }^{2}$ que celles d'Amos Barbot ${ }^{3}$ ne pallient pas ces lacunes. Des archives municipales qu'ils ont compulsées, leurs auteurs ne retiennent, année par année, que ce qui a trait aux maires, à l'activité échevinale, aux privi-

2. La Rochelle au fil des ans avec Nicolas Baudouin, t. I, XIII et XIVe siècles, publié par Favreau R. dans Archives Historiques de la Saintonge et de l'Aunis, t. LX, 2007; t. II, XVe siècle, publié par Tranchant M. dans Archives Historiques de la Saintonge et de l'Aunis, t. LXI, 2008.

3. Histoire de La Rochelle par Amos Barbot, publiée par Denys D'Aussy M. dans Archives Historiques de la Saintonge et de l'Aunis, t. XIV, XVII et XVIII, Paris Saintes, 1886, 1889, 1890. 
lèges de la ville, aux chantiers en cours. Dans toute la compilation de Barbot, ne figure qu'une seule mention de tempête, celle du 10 août 1518, vraisemblablement parce que le très grave débordement de la mer qui s'est produit ce jour-là submergea les vignes et les champs, portant ainsi un coup très rude à l'économie rochelaise alors largement tournée vers les exportations de vin et de grains.

Le médiéviste travaille donc sur des épaves. Il ne dispose, est-il besoin de le préciser, d'aucun document comparable au si précieux journal du négociant rochelais Jacob Lambertz (1733-1813) (Garnier, 2010; Garnier et Surville, 2010a). Il n'est pas pour autant totalement démuni mais il lui faut s'adonner à une collecte incertaine dans des sources très diverses, qu'il s'agisse de documents normatifs comme la coutume de l'île de Bouin énumérant les usages locaux, de cartulaires, de comptes seigneuriaux, conservés en séries très lacunaires principalement dans l'abondant chartrier de Thouars, ou encore d'un compte malheureusement unique d'exploitation salicole (Briand in Hocquet et Sarrazin, 2006). La plupart des données recueillies ont trait à la saliculture, principale activité des secteurs bas du littoral jusqu'au XIX ${ }^{\mathrm{e}}$ siècle. Obligatoirement aménagés sous le niveau des hautes mers pendant les grandes marées, les marais salants sont particulièrement exposés aux submersions marines. La circulation de l'eau salée s'effectuant par gravité des réservoirs, vasières, métières, jas, conches vers les cristallisoirs, alors uniquement des aires au sud de la Loire, toute inondation marine non seulement détruisait les monceaux du sel stocké sur les tesseliers mais interdisait toute production pour un temps plus ou moins long.

À partir de la fin du XVI ${ }^{\text {e }}$ siècle, la documentation se fait plus riche, plus dense, plus précise. Certains textes apportent un véritable récit des événements catastrophiques. C'est le cas, par exemple, du mémorial tenu à partir de 1581 par le sieur Nicolas Herpin, notaire et procureur à Saint-Martin-de-Ré ${ }^{4}$. Dans ce mémorial, Herpin ne se contente pas de mentionner; il raconte, ce qui est d'un grand prix pour évaluer la gravité des phénomènes.

Corollaire de la précédente, l'autre grande difficulté est de bien définir les phénomènes à partir d'une critique serrée des sources. Dans sa monu-

4. Médiathèque de La Rochelle, ms 163. mentale recension des tempêtes et inondations fluviales ayant affecté les Pays-Bas jusqu'au XVII siècle, Elisabeth Gottschalk insiste à juste titre sur l'absolue nécessité de faire preuve de l'esprit critique le plus aiguisé (Gottschalk, 1971, 1975, 1977). La tempête la mieux documentée n'est pas forcément la plus violente. Il en est ainsi de la moins mal connue de tout le $\mathrm{XV}^{\mathrm{e}}$ siècle. Survenue le premier dimanche de carême 1408 (4 mars en calendrier julien alors en usage soit vers le 13 mars si l'on effectue une translation en calendrier grégorien), sévissant au plus fort de midi au soleil couchant, cette tempête jette sur le rivage ou met en péril la vingtaine de « grosses heurques et autres vaisseaulx » ancrés dans la Baie entre Bouin et Le Collet ${ }^{5}$ Sarrazin in Bochaca et Sarrazin, 2007). S'est-elle accompagnée d'une submersion? Rien ne l'indique. N'eût été un différend entre Guy de Laval, sire de Retz et Catherine de Machecoul, dame de La Suze et de Bourgneuf au sujet de l'exercice du droit de bris sur un navire de Quimper, l'événement météorologique n'aurait laissé aucune trace. À l'inverse, de véritables catastrophes peuvent passer presque totalement inaperçues, telle cette submersion apocalyptique que l'on peut dater sous toute réserve de l'hiver 1351-1352 et dont on ne mesure le terrible impact qu'a posteriori : l'île de Ré fut en partie envahie par la mer (Kemmerer, 1888), les marais salants exposés de l'Olonnais furent totalement détruits ${ }^{6}$, la plaine de Barbâtre à Noirmoutier fut ennoyée pour plus d'un demi-siècle, le temps que la sédimentation fasse son œuvre (Bouhier, 1970).

Globalement, la recension des vimers pose les mêmes problèmes de critique que celle des séismes. Elle doit se défier des compilations sans références. Fort heureusement, elle n'est pas encombrée par des catalogues sans valeur tels qu'ils en existent et parfois récents pour les tremblements de terre (Alexandre in Bennassar, 1996). La démarche indispensable est de retourner aux sources originales ou aux sources de seconde main qui recopient des sources disparues.

Les mentions de submersions marines ne peuvent être collectées qu'à partir du XIVe siècle. Jusqu'alors, les sources ne fournissent aucun indice d'évé-

\footnotetext{
5. Cartulaire des sires de Rays, 1160-1449, publié par Blanchard R. dans Archives Historiques du Poitou, t. XXVIII et XXX, Poitiers, 1899, $\mathrm{n}^{\circ}$ CCLVII.

6. Cartulaire de l'abbaye d'Orbestier, publié par La Boutetiere L. de, dans Archives Historiques du Poitou, t. VI, Poitiers, 1877, n 194.
} 
nements catastrophiques, à une exception près, notable, très difficile à analyser, le récit placé au début de la coutume de Bouin d'un «vimer » atypique, vraisemblablement un tsunami. Rédigée en 1644 sur la base d'un texte de 1421, la coutume relate que le 7 juin 567, par un temps calme, la mer s'éleva soudainement à une hauteur telle qu'elle submergea l'île entière et noya tous les habitants ${ }^{7}$. À la suite de cette catastrophe, l'île resta déserte pendant environ 10 ans. La situation politique décrite, parfaitement anachronique pour le VI $\mathrm{I}^{\mathrm{e}}$ siècle, de même que les noms tout aussi anachroniques des colons qui reprirent possession du territoire interdisent d'accorder le moindre crédit à la date indiquée. Tout se passe comme si la mémoire d'un tsunami venait se fondre dans un récit légendaire des origines de l'île. Qu'il soit provoqué par un tremblement de terre au niveau de la dorsale médio-atlantique ou par un effondrement au bord du plateau continental, un tsunami est en effet possible sur la côte atlantique mais sans atteindre la proportion de vagues gigantesques susceptibles de s'abattre sur les côtes de l'Asie du Sud-Est. Les phénomènes du type mascaret enregistrés à La Rochelle le 9 juin 1875, le 22 avril 1882 et le 24 décembre 1892 en apportent une preuve tangible (Garnier et Surville, 2010b).

Avant 1500, une recension comparable à celle qu'a effectuée aux années 1970 E. Gottschalk pour les Pays-Bas est impossible à réaliser (Gottschalk, 1970, 1975). Les sources sont trop lacunaires et les problèmes de datation trop aigus. Toute approche sur la fréquence des phénomènes est vouée à l'échec. Il est en particulier impossible de savoir s'il y a eu une recrudescence d'événements catastrophiques dans les années 1330-1340 comme en mer du Nord. De même, tenter d'appliquer l'indice de sévérité des tempêtes tel que le climatologue $\mathrm{H}$. Lamb l'a formulé (V3 x Amax x D; vitesse du vent au cube $\mathrm{x}$ l'aire maximale des dommages $\mathrm{x}$ durée du phénomène) est évidemment hors de portée (Lamb, 1991).

Le tableau des "vimers » proposé ci-dessous (tableau 1, figure 1) n'est qu'une esquisse provisoire. D'autres informations collectées dans des sources non dépouillées viendront le compléter. Constituer une banque de données sur toutes les tempêtes et submersions ayant affecté le littoral atlantique du

7. Coutume de l'île de Bouin publiée par Luneau M. et Gallet E., Documents sur l'île de Bouin, Nantes, 1874, pièce justificative $\mathrm{n}^{\circ} \mathrm{I}$.
$\mathrm{XIV}^{\mathrm{e}}$ siècle à nos jours est une entreprise éminemment souhaitable. Elle impliquera nécessairement des collaborations nombreuses.

\section{LES POPULATIONS LITTORALES FACE AUX DÉBORDEMENTS DE LA MER}

Les populations littorales étaient, faut-il le rappeler, majoritairement paysannes. Deux de leurs activités étaient particulièrement exposées aux inondations marines, la saliculture et l'agriculture des relais, autrement dit des laisses de mer.

L'importance majeure de la saliculture n'est plus à démontrer. Sur tout ce littoral, une large part des schorres mûrs était aménagée en marais salants, lesquels constituaient, avec ceux de Setubal et Aveiro au Portugal, les principales zones exportatrices de sel marin vers l'Europe du Nord (Hocquet et Sarrazin, 2006). Autour de la Baie, les chiffres de 200000 à 250000 aires saunantes aux années 1340 et de plus de 250000 voire 3000000 à la fin du $\mathrm{XV}^{\mathrm{e}}$ siècle après la dépression de 1350-1450 ont pu être avancés (Sarrazin, 1999, 2005). À elle seule, l'île de Bouin compte 66857 aires en 1527. Pour les marais charentais, l'intendant Bégon donne en 1698 une statistique de 32266 livres (une livre = 20 aires) soit 645320 aires dont 21878 livres en exploitation soit 437560 aires, Seudre et Brouage représentant 23300 livres soit 466000 aires (Delafosse et Laveau, 1960). Et, selon toute vraisemblance, la capacité productive n'a pas beaucoup évolué entre le $\mathrm{XVI}^{\mathrm{e}}$ et la fin du XVII ${ }^{\mathrm{e}}$ siècle. Dans tous ces secteurs, la hantise des propriétaires et des exploitants était de voir un « vimer » ou « ondacion d'ayves » engloutir en quelques heures les bassins et les cristallisoirs aménagés par des générations de sauniers.

Quant aux relais, ces schorres conquis sur la mer, l'attrait qu'ils exerçaient par la richesse de leur sol est évident. Le long du Dain, ce bras de mer qui séparait l'île de Bouin du continent, ils constituaient aux années 1340 une composante non négligeable des domaines de petits seigneurs. Plus au sud, une série assez homogène de 139 aveux et dénombrements remis au seigneur de Champagné-les-Marais entre 1528 et 1561 et copiés dans un terrier du $\mathrm{XVIII}^{\mathrm{e}}$ siècle ${ }^{8}$ permet de mesurer leur importance le

8. Archives départementales de la Vendée, 1 E 440 


\begin{tabular}{|c|c|c|}
\hline $\begin{array}{l}\text { Date } \\
\text { En calendrier julien jusqu'au } 4 \text { octobre } \\
1582 \text { puis en calendrier grégorien à partir } \\
\text { du } 15 \text { octobre } 1582\end{array}$ & Source & Eléments connus de l'événement \\
\hline Hiver 1351-1352? & $\begin{array}{l}\text { Mentions a posteriori dans le cartulaire } \\
\text { d'Orbestier (n 194), dans le chartrier } \\
\text { de Thouars (AN, 1 AP } 1974 \text { n}^{\circ} 50 ; 1 \text { AP } \\
\left.1976 \text { n }^{\circ} 167\right) \text {, indication sans référence } \\
\text { dans l'ouvrage de Kemmerer sur l'île de } \\
\text { Ré }\end{array}$ & $\begin{array}{l}\text { Submersion majeure à Ré, en Olonnais, à } \\
\text { Noirmoutier }\end{array}$ \\
\hline 4 mars 1408 (n. st.) & Cartulaire des sires de Retz ( ${ }^{\circ}$ CCLVII) & $\begin{array}{l}\text { Très violente tempête sur la Baie entre } \\
\text { midi et la nuit. Navires jetés à terre entre } \\
\text { Bouin et Le Collet. Pas d'indication de } \\
\text { submersion }\end{array}$ \\
\hline 27 janvier 1469 (n. st.) & $\begin{array}{l}\text { Chartrier de Thouars, compte de la } \\
\text { seigneurie de Bouin (AN, } 1 \text { AP 2132) }\end{array}$ & $\begin{array}{l}\text { Île de Bouin partiellement inondée } \\
\text { (victimes) }\end{array}$ \\
\hline Avant juillet 1470 & $\begin{array}{l}\text { Carnet de comptes des Blanchet (AD } \\
\text { Loire-Atlantique } 2 \text { E 382) }\end{array}$ & $\begin{array}{l}\text { Submersion catastrophique de tesseliers } \\
\text { vers Prigny (Baie) }\end{array}$ \\
\hline Hiver (?) 1492-1493 & Chartrier de Thouars (AN, l AP 1964) & Chaussée rompue à Noirmoutier \\
\hline 1507 & Chartrier de Thouars, (AN, 1 AP 2077) & $\begin{array}{l}\text { Large brèche dans les murailles ouest } \\
\text { de Royan provoquée par «la grande } \\
\text { impetuosité et tormente de la mer» }\end{array}$ \\
\hline Hiver $1509 / 1510$ & $\begin{array}{l}\text { Cahiers de compte de l'abbaye de Buzay } \\
\text { (AD Loire-Atlantique, H 28); exemption } \\
\text { fiscale de } 5 \text { ans aux habitants de Bouin } \\
\text { délivrée par Anne de Bretagne (Luneau } \\
\text { et Gallet, pj XIII) }\end{array}$ & $\begin{array}{l}\text { Série de submersions consécutives à des } \\
\text { tempêtes en lîle de Bouin }\end{array}$ \\
\hline 10 août 1518 & Amos Barbot, Histoire de La Rochelle & $\begin{array}{l}\text { "1518-Au moys d'aoust de ladite année, le } \\
\text { jour de la saint Laurent, la mer se desborda } \\
\text { aux costes de cette ville, qui causa une } \\
\text { perte incroyable, gastant toutes les vignes } \\
\text { et champs" }\end{array}$ \\
\hline Mercredi 22 août 1537 & Chartrier de Thouars, (AN 1 AP 2002) & $\begin{array}{l}\text { Ré partiellement submergée; les bossious } \\
\text { des marais salants sont ennoyés; le sel } \\
\text { est perdu; le village de La Couarde } \\
\text { est inondé (eau jusqu'au plancher de } \\
\text { plusieurs maisons) }\end{array}$ \\
\hline Entre le 30 mars et le $1^{\text {er }}$ avril 1579 & $\begin{array}{l}\text { AN Q }{ }^{1} 130 \text { n }^{\circ} 3 \text { et AD Charente- } \\
\text { Maritime G } 253 \text { n }^{\circ} 4 \text { (Périsse, 2011) }\end{array}$ & $\begin{array}{l}\text { Ouverture du chenal Madame en l'île } \\
\text { d'Oléron }\end{array}$ \\
\hline 10 juin 1584 & $\begin{array}{l}\text { Mémorial du notaire Herpin, } \\
\text { Médiathèque La Rochelle, ms } 163\end{array}$ & $\begin{array}{l}\text { Très violente tempête sur la côte. } \\
\text { Céréales « brûlées ». Submersion? }\end{array}$ \\
\hline 11 décembre 1589 & Mémorial du notaire Herpin & Très violente tempête sur la côte \\
\hline 24 février 1591 & Mémorial du notaire Herpin & $\begin{array}{l}\text { Submersion de l'île de Ré, divisée comme } \\
\text { originellement en trois îles. Marais } \\
\text { salants détruits. }\end{array}$ \\
\hline 4 octobre 1591 & Mémorial du notaire Herpin & $\begin{array}{l}\text { Submersions à Bouin, Ré, Brouage, } \\
\text { Marennes, Oléron. Vimer plus grave que } \\
\text { celui du } 24 \text { février }\end{array}$ \\
\hline 31 décembre 1598 & $\begin{array}{l}\text { Mémorial du notaire Herpin } \\
\text { Supplique des habitants de Bouin à Louis } \\
\text { XIII (30 juin 1627) (Luneau et Gallet, pj } \\
\text { XXXIII) }\end{array}$ & $\begin{array}{l}\text { Submersion majeure à Bouin et à Ré. } \\
\text { L'île de Bouin est évacuée. Destruction } \\
\text { catastrophique de marais salants. }\end{array}$ \\
\hline
\end{tabular}

Tableau $1:$ «Vimers » ayant touché le littoral atlantique (AN : Archives nationales; AD : Archives départementales; ms : manuscrit; pj : pièce justificative)

"Vimers" that affected the Atlantic coast 


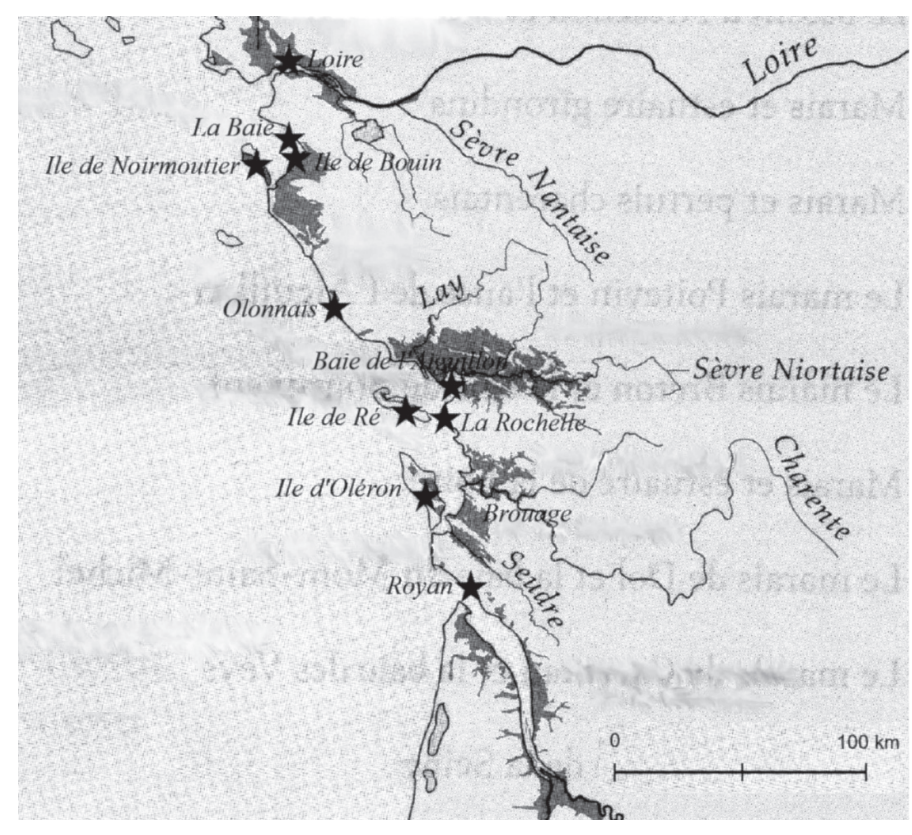

Figure 1 : Localisation des secteurs littoraux touchés par les vimers mentionnés dans les sources écrites

tioned in written documents

long de la baie de L'Aiguillon. Sur ces 139 actes, 32 mentionnent de un à cinq relais. En outre, les indications fournies par les confronts montrent que de nombreux seigneurs, presque tous les établissements religieux, abbayes, prieurés mais aussi les confréries, les curés détenaient ces terres riches (Guibot, 2002).

Le point commun de la saliculture et de l'agriculture de relais était de mettre en valeur des terres situées sous le niveau des hautes mers lors des malines (marées de vive eau). Face à la menace clairement perçue comme permanente de l'invasion marine, la préoccupation majeure des sociétés littorales était de se protéger, en édifiant des levées, des chaussées de mer, des bots de garde. En ce temps, il n'était point de hautes digues défensives ou offensives dressées en front de mer. Au reste, le terme thiois-flamand de digue n'apparaît que tardivement dans toute la région. Employé pour la côte poitevine dans les plaidoiries du Parlement de Paris et fort épisodiquement par la Chancellerie royale, a-t-il été vulgarisé par les ingénieurs néerlandais lors des grands travaux du XVII ${ }^{\mathrm{e}}$ siècle? L'hypothèse serait à vérifier. Quoi qu'il en soit, il n'existait pas de système défensif réellement communautaire, d'institutions comparables aux wateringues de Flandre. La construction et l'entretien des chaussées relevaient des détenteurs du sol.

Les sources permettent d'effectuer, à ce sujet, deux études de cas portant sur des zones sensibles déjà évoquées, la baie de L'Aiguillon et l'île de Bouin. Le secteur nord-est de la baie de L'Aiguillon est éclairé à la fois par une carte ancienne tant bien que mal dessinée lors d'un partage en 1656 (figure 2) et par un important dossier documentaire formé, en 1526-1527, à l'occasion d'une réhabilitation d'un bot de garde en front de mer.

Orientée vers le sud, la carte de 1656 présente plusieurs centres d'intérêt. Elle montre en premier lieu la progression des terres entre le début du XvI ${ }^{\mathrm{e}}$ siècle et le milieu du XvII ${ }^{\mathrm{e}}$ siècle. Dans les années 1520 , la mer venait encore clapoter, d'après le témoignage des textes, jusqu'au bot de garde. En 1656, elle n'arrive même plus jusqu'à la digue qui a été construite depuis entre l'embouchure de la Sèvre (en haut à gauche) et le canal de la Charrie ou de Luçon (en haut à droite). Des relais de mer sont en effet indiqués au sud de cette digue. Par ailleurs, la carte figure clairement les différents achenaux qui se jettent dans la baie de L'Aiguillon et à partir desquels était organisé l'entretien du bot de garde. Enfin, elle montre comment la succession des bots, bots de garde, bots herbus, bots cheminaux constituait un dispositif efficace de protection face à une éventuelle submersion marine.

Quant au dossier documentaire constitué par un mandement du roi François I ${ }^{\text {er }}$, un procès-verbal de visite et la liste des propriétaires astreints aux réfections, il apporte beaucoup à la connaissance du mode social de construction et d'entretien des bots de garde en la châtellenie de Champagné au premier XvI ${ }^{\mathrm{e}}$ siècle. Dans les années 1510-1520, l'état des marais de la Sèvre niortaise et des marais littoraux paraît s'être dégradé. Était-ce une conséquence des «vimers » de 1509-1510? On ne saurait l'affirmer. L'État monarchique intervient comme il le fait depuis le règne de Charles VI. Par un mandement à son sénéchal de Poitou, en date du 11 août 1526, François Ir enjoint de rechercher "tous évesques, abbéz et autres gens d'Église, nobles, rosturiers... à faire ou faire faire réparation ». Se faisant manifestement l'écho de doléances exprimées par les populations, l'acte dresse un tableau bien sombre des lieux : "Mesmement par deffault d'entretenir certaines levéez ou botz appelez les botz des 


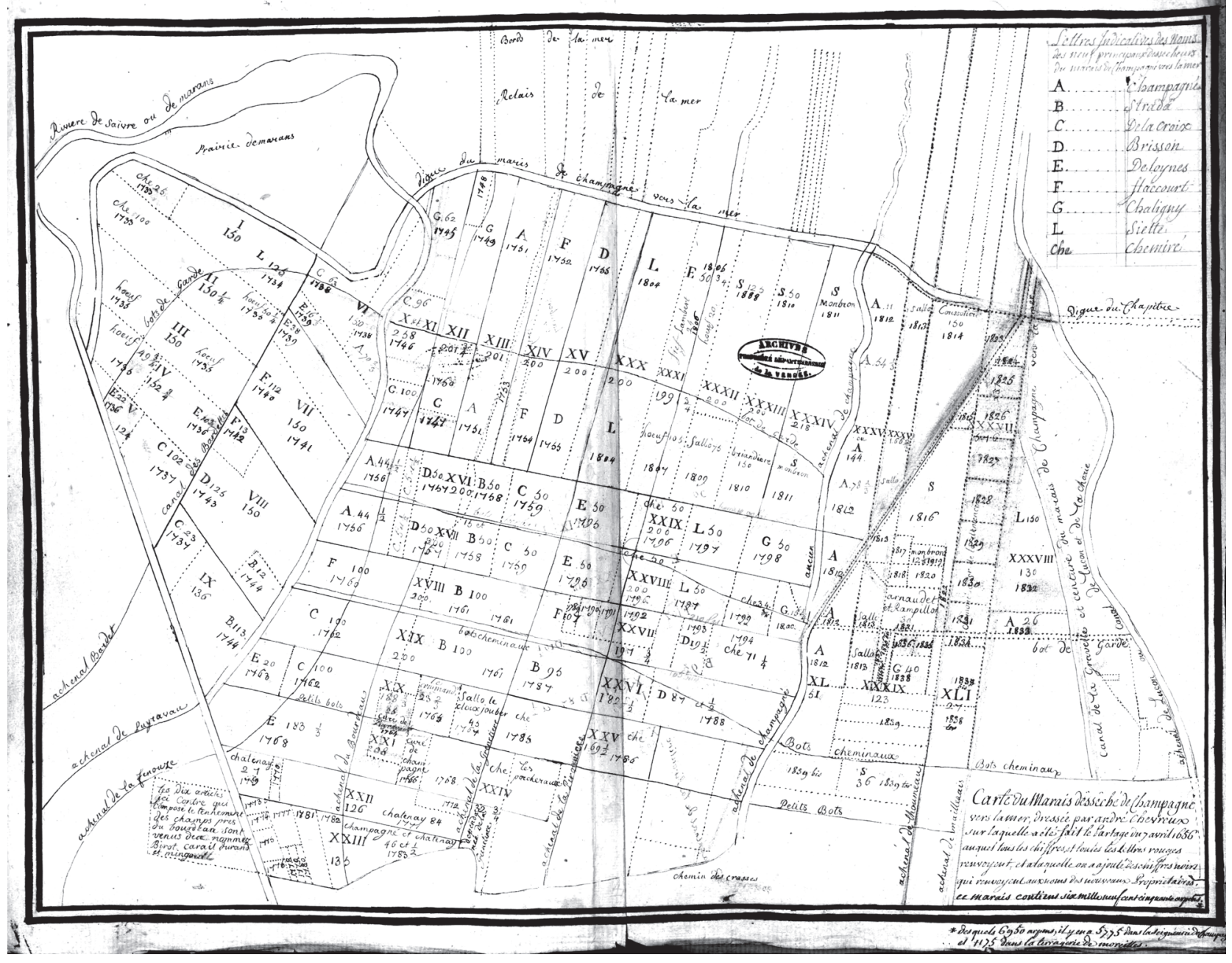

Figure 2 : Carte du marais de Champagné vers la mer dressée par André Chevreux en 1656 (Archives départementales de la Vendée, 1 E 442) Map of the marsh to the sea Champagné drawn by André Chevreux in 1656 (Archives départementales de la Vendée, 1 E 442)

rellays contre la mer qui, par chacun jour ou quequesoit souvent noye, submerge, pert et gaste totalement ledit païs et le rend stérile et presque inhabitable9." Le procès-verbal de visite, issu d'une enquête effectuée entre le 7 mars et le 21 août 1527, est beaucoup plus explicite. Il précise que la construction et l'entretien des bots de protection face à la mer incombaient à ceux qui détenaient des «dommaines qu'ilz avoient contiguz esdits bots et achenaulx et es environs ${ }^{10}$ ", en d'autres termes aux propriétaires dont les domaines touchaient aux bots mais aussi à ceux qui, en amont, égouttaient leurs terres dans les achenaux coulant vers la baie de L'Aiguillon et qui avaient aussi intérêt à la protection de tout le sec-

9. Clouzot E., Les marais..., pièce justificative $n^{\circ}$ XIII

10. Ibidem, pièce justificative $\mathrm{n}^{\circ} \mathrm{XIV}$. teur littoral. Ainsi est dressée une liste des établissements ecclésiastiques, des nobles et des roturiers appelés à relever les bots de garde de la châtellenie de Champagné entre l'achenal de la Charrie et celui de la Coueresse avec le nombre de toises assignées à chacun ${ }^{11}$. De cette liste, l'on retiendra le grand nombre de propriétaires concernés, 46 au total pour une longueur minimale de 3775 toises (environ 6800 mètres) à réparer. Sur les 43 qui se sont vus assigner un ou des tronçons précisément mesurés, 24 soit $55,8 \%$ devaient relever 20 toises (36 mètres) ou moins. À lui seul, un gros fermier, manifestement un entrepreneur de relais, Colas Siméon, prenait en charge 1 819,6 toises (3275 mètres), près de la moi-

11. Ibid., pièce justificative $\mathrm{n}^{\circ} \mathrm{XV}$. 
tié du bot. Les tableaux 2 et 3 montrent clairement la principale faiblesse du dispositif : l'émiettement du chantier. Délibérée ou non, l'incurie de quelques propriétaires à colmater les brèches pouvait ruiner les efforts des autres. Le risque était d'autant plus effectif qu'aucune autorité ne coordonnait l'opération. L'État se bornait, en l'espèce, à rappeler les détenteurs de terre à leurs devoirs.

Aucun document n'apporte d'informations sur les travaux réellement effectués. L'opération a dû se révéler d'autant plus délicate à conduire que le dispositif de desséchement des marais et de protection contre la mer présentait un élément majeur de fragilité au niveau des portes qu'un texte légèrement postérieur décrit en ces termes «lesquels eschenaulx avoient leurs portereaux aux bots de garde devers la mer pour les faire couller en icelle, lesquels portereaux fermoient si et quand la marée venoit jucques au pied desdits botz et levée de garde ${ }^{12} »$.
Autre excellent point d'observation, l'île de Bouin, au fond de la Baie, offre une documentation à la fois normative et en prise avec la réalité, principalement des comptes seigneuriaux du sire de Retz et des cisterciens de Buzay disponibles par bribes à partir de 1468 (Sarrazin, 2005, 2008). Formée autour d'un îlot calcaire sur lequel est construit le village, "circuitée et environnée de mer, subjecte à estre submergée par les innondations, grandeur et abondance de la mer ", elle n'est au fond qu'un immense schorre émergé de l'océan; son extrême platitude facilite grandement l'aménagement des marais salants mais constitue un facteur évident de risque face à la «malice » de l'océan. Située sous le niveau de la haute mer lors des malines, elle était protégée par tout un dispositif de chaussées édifiées face à la mer et le long des étiers. Citées depuis les débuts de la documentation au XIII ${ }^{\mathrm{e}}$ siècle mais évidemment plus anciennes,

\begin{tabular}{|c|c|c|c|c|c|c|c|}
\hline Achenal contigu & La Coueresse & Puyraveau & La Fenouze & La Grénetière & La Pironnière & Champagné & L'Hommeau \\
\hline $\begin{array}{c}\text { Nombre de toises } \\
\text { par achenal }\end{array}$ & 205 & 1551 & 609 & 225 & 326 & 540 & 339 \\
\hline $\begin{array}{c}\text { Nombre de } \\
\text { propriétaires } \\
\text { concernés }\end{array}$ & 8 & 3 & 18 & 1 & 18 & 13 & 18 \\
\hline $\begin{array}{c}\text { Fourchette du } \\
\text { nombre de toises } \\
\text { de bot à relever } \\
\text { assignées à chacun }\end{array}$ & $6-60$ & $25-1500$ & $4-100$ & 225 & $6-60$ & $5-291$ & $4-108$ \\
\hline
\end{tabular}

Tableau 2 : La réfection du bot de garde « estant au devant la mer» en la châtellenie de Champagné entre l'embouchure de l'achenal de la Coueresse (ou de la Bardette) et l'embouchure de l'achenal de la Charrie [1527]. La toise utilisée n'étant pas précisée, l'on conviendra d'appliquer l'équivalence 1 toise $=1,8$ mètre.

The rehabilitation of the bot de garde (embankment) "by the sea" in the châtellenie (manor) of Champagné between the mouth of the canal Coneresse and the mouth of the canal Charrie [1527]. The toise used is not specified; one can apply the equivalence 1 toise $=1.8$ meter

\begin{tabular}{|c|c|}
\hline $\begin{array}{c}\text { Nombre de toises à relever par propriétaire. } \\
\text { Un même propriétaire peut réparer le bot à différents endroits, } \\
\text { relevant de plusieurs achenaux }\end{array}$ & Nombre de propriétaires \\
\hline$\leq 5$ & 9 \\
\hline$>5-10$ & 8 \\
\hline$>10-20$ & 7 \\
\hline$>20-50$ & 9 \\
\hline$>50-100$ & 3 \\
\hline$>100-500$ & 6 \\
\hline$>1500$ & 1 \\
\hline
\end{tabular}

Tableau 3 : Répartition des réparations par propriétaire repair among owners

12. Ibid., pièce justificative $n^{\circ} \mathrm{XVII}$. 
les chaussées de mer, de tracé discontinu étaient majoritairement situées à l'ouest et au sud-ouest de l'île. Les réparations mentionnées au chapitre des mises dans les comptes de la seigneurie (1468-1469) ou dans ceux de l'abbaye de Buzay (1505-1512) apportent d'utiles précisions sur leur construction. Toutes étaient faites de bri amoncelé, consolidé par des galets et des pierres. Édifiées sur des schorres, elles ne paraissent pas avoir été d'une grande hauteur à la différence des futures digues construites sur des slikkes au temps des polders (Verger, 1968, 2005). Il faut attendre les registres des chaussées datés de 1600 et 1662 pour disposer d'indications chiffrées. La hauteur habituelle était alors de 7 pieds soit environ 2,3 mètres (Baron, 1994). Intéressante en elle-même, la donnée permet également d'évaluer tant bien que mal la surcote de la mer lors d'un « vimer ». Un dépassement de chaussée signalé à 3 pieds équivaut à une surcote d'environ un mètre.

Les chaussées internes étaient élevées avec les vases de curage des étiers. Elles contribuaient tout autant à la protection de l'île. En d'autres termes, face au risque d'inondation marine, les populations avaient mis en place non pas un système de fortes digues défensives; elles n'en avaient ni les moyens techniques ni la conception. Elles ont édifié, au fur et à mesure, un réseau complexe de levées médiocres se fondant dans le paysage salicole (Sarrazin, 2005). L'efficacité d'une telle protection était relative. L'inondation marine de mauvaise saison paraît avoir été sinon habituelle du moins fréquente. L'ingénieur-cartographe Claude Masse note encore au début du XVIII ${ }^{\mathrm{e}}$ siècle que "l'isle est si basse que plusieurs fois les habitants ont eu de l'eau jusqu'aux genoux dans leurs maisons et ne pouvaient faire $d u$ feu que du four. Cela arrive souvent quand les digues rompent » (Pawlowski, 1911).

Si la gestion des infrastructures d'utilisation communautaire, les ports, les passages, les ponts ressortissait aux prérogatives de la seigneurie concédées aux habitants organisés au sein de la fabrique (Sarrazin, 2005), l'édification et l'entretien des chaussées revenaient, comme à Champagné, aux détenteurs des terres contiguës. La coutume de Bouin accorde une grande place aux questions de maintenance, notamment des éléments de fragilité qu'étaient les coués (conduits sous les levées). Qui en utilisait ou en déplaçait un devait tenir en bon état l'endroit sur 5 brasses de hauteur, longueur et largeur. En cas de rupture, le détenteur avait l'obligation de construire une levée de colmatage derrière l'ouverture afin d'empêcher l'eau salée d'envahir le marais. Les comptes disponibles fournissent de précieux renseignements sur les pratiques. La donnée essentielle qui ressort de leur analyse, c'est la facilité à embaucher de la maind'œuvre à très bon marché. Il y avait pléthore de journaliers et relever une chaussée n'exigeait pas une grosse qualification. Dans ces conditions, le coût d'entretien des chaussées était très faible. Pour les deux années 1468 et 1469, il s'élève en ce qui concerne les chaussées que devaient entretenir le sire de Retz à 4,13\% des dépenses de la seigneurie (Sarrazin, 2005). Le même constat peut être fait pour les réparations de cinq chaussées des cisterciens de Buzay après les tempêtes de 1510. Ainsi, à la condition qu'il ne détruisît pas complètement les salines, un « vimer » pouvait constituer une aubaine pour le petit peuple des manouvriers en induisant une distribution de salaires. Il y a là une clé de compréhension du mode de protection. Faites de bri et de pierres, les chaussées étaient au fond assez fragiles. Elles rompaient à la première survenue d'un vimer mais elles étaient faciles à réparer. Leur possible rupture était une donnée assimilée, intégrée au mode de fonctionnement de la société. Se trouve ainsi révélée une attitude face au danger qui diffère profondément de ce qu'elle est aujourd'hui.

\section{LA MÉMOIRE VIVANTE DES RISQUES MARINS}

Qu'elles aient eu à faire face à une descente hostile d'Anglais, d'Espagnols, de Néerlandais ou à une montée des eaux, les paysanneries maritaines étaient en permanence sur le qui-vive. Elles épousaient le danger pour mieux le conjurer. Populations en frontière de mer, elles se considéraient ellesmêmes comme la protection du pays, un rempart vivant s'opposant aux ennemis du pays et à la «malice» de l'océan (Mollat, 1983). Elles utilisaient fréquemment, au reste, l'argument pour obtenir des privilèges du roi. Elles savaient mettre en avant leur comportement rebelle et leur capacité de résistance aux menaces naturelles. "Dangereuses gens qui souventes fois font désobéissance » dit le mémoire de 
1451 en guise d'avertissement au conseil du roi ${ }^{13}$. Face à la violence de la mer systématiquement perçue comme un horizon menaçant, elles ont développé une remarquable aptitude à la défense. C'est cet état d'esprit que révèlent les interrogatoires des séditieux de Beauvoir-sur-Mer et de ses environs en 1480 après une révolte contre une augmentation de la fiscalité étatique du sel. Un certain Briend Lamynais de Bois-de-Cené, auteur de coups sur les sergents du roi et délivré en chemin vers minuit par des gens armés de son village explique ainsi que " pour la craincte desdits Espaigneulx, Angloys, Hollandays et autres gens de mer qui chacun jour et nuyt viennent piller et robber les pauvres gens demourans sur la coste de la mer dudit bas Poitou, ilz ont acoustumé pour résister et eulx en donner garde de faire toutes les nuys le guet affin si aucune chose survient de crier alarme et faire assembler les gens $d u$ pays $^{14}{ }^{1}$.

Les faibles capacités techniques de ces sociétés contribuaient à mettre le danger marin au cœur de la vie. Qu'étaient les chaussées devant Brouage, à Ré, à Bouin? On l'a dit; de simples talus de terre de bri plus ou moins bien renforcés de galets. Les bots de garde en baie de l'Aiguillon n'étaient guère différents. De tels barrages étaient d'autant plus précaires que sous l'action du soleil, de la pluie et de la mer, le bri se désagrège facilement. Chaque hiver, la «fortune de la marée » arasait ainsi tel ou tel tronçon. L'inondation était donc habituelle. La rupture fréquente des levées entretenait la mémoire vivante, permanente de l'inondation, alors que la puissante digue contemporaine, surtout si le marais en arrière a été desséché, favorise l'oubli.

La mémoire des « vimers » devait sans nul doute beaucoup à la tradition orale, dont les délégués de la ville de Poitiers se font l'écho en 1451 en s'adressant au conseil du roi Charles VII. Elle revêtait par ailleurs une autre forme, écrite celle-ci, plus savante, plus intéressée également dans la mesure où elle servait à obtenir des privilèges, que ce fût une exemption fiscale ou une dispense d'entretien des gens de guerre. Dans leur dialogue avec l'État, seigneurs et populations du littoral mettaient en avant, sur la base de textes dûment conservés, deux menaces principales, les descentes ennemies et les submersions marines. Une nouvelle fois, l'île de

13. Mémoires, supra note 1

14. Bibliothèque nationale de France, manuscrit français 2896, fo $32 \mathrm{r}^{\circ}$
Bouin est la mieux documentée, à travers différents actes porteurs de franchises. Les lettres royales de novembre 1466, octroyant l'exemption de toutes tailles et aides aux habitants, synthétisent ainsi en ces termes lapidaires les résultats d'une enquête diligentée 14 ans auparavant : "Laquelle isle fut ou temps passé recouvrée par industrie et puissance de chaussées, et pour icelle garder tant de inundacions de la mer que des périlz de noz anciens ennemis les Anglois, lesdiz habitans, supplians, ont fait et font chacun jour de très grans mises et despenses, peines et travaulx tellement que à peine les pevent supporter $^{15}$. » Une quarantaine d'années plus tard, par un acte en date du 23 juin 1511, Anne, duchesse de Bretagne et reine de France exempte pour cinq ans les manants de l'île de toute imposition «à cause des grandes et excessives eaux et marées qui en l'année 1509 [8 avril 1509-30 mars 1510] surmontèrent les levées et chaussées faictes pour la deffense et conservation de ladite isle, tellement que ycelle isle fut submergée, les moulins y estant et lesdites levées et chaussées rompues, démolys et abattues ${ }^{16}{ }$. C'est un véritable bilan des principaux «vimers » ayant touché leur île sur 128 ans que présentent un siècle plus tard les habitants dans leur supplique à Louis XIII le 30 juin 1627, afin d'obtenir la dispense d'entretenir des hommes d'armes : «Au subject des desbordemens et des vimers généraux dont ils se sont veus accabléz depuis l'an mil cinq cens de plus de quinze et contrains en deux d'iceulx, notamment en l'an mil cinq cens quatre vingt dix huict de quitter et abandonner l'isle qu'ils awvoient peu de jour après reprinse et d'icelle réédifié les digues et chaussées qu'ils ont maintenu par leur travail continuel ${ }^{17}$... » Il était malheureusement exclu qu'une simple supplique précisât les dates des grandes tempêtes; la seule année mentionnée est 1598 , celle où s'est produit un événement cataclysmique particulièrement traumatisant pour la population.

\section{Conclusion}

Dans l'histoire des submersions marines ayant affecté le littoral atlantique, la période $\mathrm{XIV}^{\mathrm{e}}-\mathrm{XVI}^{\mathrm{e}}$ siècles

15. Recueil des documents concernant le Poitou contenus dans les registres de la Chancellerie de France, t. XI publié par Guérin P. dans Archives Historiques du Poitou, t. XXXVIII, Poitiers, 1909, n MCCCCXXIV, p. 62 .

16. Luneau M. et Gallet E., Documents..., pièce justificative ${ }^{\circ}$ XIII. 17. Ibidem, pièce justificative $\mathrm{n}^{\circ} \mathrm{XXXIII}$ 
marque donc la sortie du silence documentaire. Notre connaissance des événements météorologiques, de leur déroulement, de leur impact sur la vie des populations reste néanmoins très imparfaite, étroitement liée qu'elle peut être aux aléas de la production et de la conservation des sources mais encore aux attitudes des sociétés face à la mort. À une mention près, furtive dans un compte, les informations relatives aux victimes sont inexistantes. La notion de « bilan humain », aujourd'hui prioritaire dans l'annonce d'une catastrophe, était largement étrangère à la mentalité du temps. Quant aux secours que pouvaient mettre en œuvre les communautés littorales touchées par telle ou telle tempête, il est impossible d'en donner la moindre idée au-delà de quelques témoignages généraux de solidarité. Connaître par exemple les circonstances dans lesquelles se sont déroulées les deux évacuations de l'île de Bouin au $\mathrm{XVI}^{\mathrm{e}}$ siècle est évidemment hors de portée.

De tous les «vimers » qui se sont effectivement produits, trois ou quatre peuvent retenir l'attention. Vraisemblablement apocalyptique, inondant durablement de larges secteurs des îles, celui de l'hiver 1351-1352 (?) a porté un coup sévère à l'agriculture et à la saliculture; ses stigmates sont encore repérables plus d'un demi-siècle après l'épisode; il est venu s'ajouter aux autre fléaux du temps, la peste et la guerre. Les tempêtes de 1509-1510 sont également remarquables par les destructions de chaussées et d'infrastructures. La grande submersion de la Saint-Laurent 1518 a frappé les esprits tant par son importance que par la saison où elle est survenue. Quant à l'incroyable tourmente du 31 décembre 1598, aggravée par la tempête du 3 janvier suivant, elle a été vécue comme un traumatisme majeur dont l'écho s'est diffusé le long du littoral; c'est le notaire Herpin de Saint-Martin-de-Ré qui signale que plus de 25 navires ont été perdus en Loire.

Face au risque marin vécu comme permanent, les sociétés paysannes du littoral se sont adaptées par la force des choses. Elles ne disposaient pas des moyens techniques pour s'opposer de front au péril de la mer; elles n'en avaient pas non plus la conception. Elles épousaient le danger marin, l'assumaient, n'exprimaient de vraies doléances qu'à l'occasion de «vimers » exceptionnels. Dotées d'une mémoire vivante des tempêtes et des submersions marquantes, elles intégraient ces grands dérangements dans leur mode d'existence.

\section{Bibliographie}

Baron B., 1994. L'̂́le de Bouin dans la première moitié du XVII ${ }^{e}$ siècle, Mémoire de maîtrise, Université de Nantes, $147 \mathrm{p}$.

Bennassar B. (dir.), 1996. Les catastrophes naturelles dans l'Europe médiévale et moderne, Flaran XV, Toulouse, Presses Universitaires du Mirail, $272 \mathrm{p}$.

Bochaca M., Sarrazin J.-L. (dir.), 2007. Ports et littoraux de l'Europe atlantique. Transformations naturelles et aménagements humains (XIV -XVI siècles), Rennes, Presses Universitaires de Rennes, $262 \mathrm{p}$.

Bouhier C., 1970. Les possessions de l'abbaye Blanche à Barbâtre, Lettres aux amis de Noirmoutier, $2^{\text {nde }}$ série, $\mathrm{n}^{\circ} 1$, p. 3-10.

Clouzot E., 1904. Les marais de la Sèvre niortaise et du Lay du $x^{e}$ à la fin $d u \mathrm{XVI}^{e}$ siècle, Paris Niort, Champion H., Clouzot L. (réimpression Laffitte, 1979), 283 p.

Delafosse M., Laveau C., 1960. Le commerce du sel de Brouage aux XVII et XVIII siècles, Paris, Armand Colin, 134 p.

Garnier E., 2010. Les dérangements du temps. 500 ans de chaud et de froid en Europe, Plon, Paris, 245 p.

Garnier E., Surville F. (dir.). 2010a. Climat et révolutions autour du journal du négociant Jacob Lambertz (1733-1813), Saintes, Le Croît vif, 576 p.

Garnier E., Surville F. (dir.), 2010b. La tempête Xynthia face à l'histoire. Submersions et tsunamis sur les littoraux français $d u$ Moyen Âge à nos jours, Saintes, Le Croît Vif, 174 p.

GotTschalk M. K. E., 1971. Stormvloeden en rivieroverstromingen in Nederland, tome I, de periode voor 1400, Assen, Van Gorcum § Comp., 581 p.

GotTschalk M. K. E., 1975. Stormvloeden en rivieroverstromingen in Nederland, tome II, de periode1400-1600, Assen, Van Gorcum § Comp., 880 p.

GotTschalk M. K. E., 1977. Stormvloeden en rivieroverstromingen in Nederland, tome III, de periode 1600-1700, Assen, Van Gorcum \$ Comp., 440 p.

Guibot E., 2002. La châtellenie de Champagné-les-Marais (1442-1561), Mémoire de maîtrise, Université de Nantes, 2 vol., 215 p. et 217 p.

Hocquet J.-C., Sarrazin J.-L. (dir.), 2006. Le sel de la Baie. Histoire, archéologie, ethnologie des sels atlantiques, Presses Universitaires de Rennes, Rennes, $411 \mathrm{p}$.

Kemmerer Dr Th. E., 1888. Histoire de l'île de Ré. L'Insula Rhéa, Saint-Martin-de-Ré, imprimerie Jeanne d'Arc, $2^{\text {nde }}$ édit., 540 p.

Lamb H., 1991. Historic Storms of the North Sea, British Isles and Northwest Europe, Cambridge, Cambridge University Press, 204 p.

Mollat M., 1983. La vie quotidienne des gens de mer en Atlantique, IX $X^{e}-X V I^{e}$ siècle, Paris, Hachette, 261 p.

Pawlowski A., 1911. Les transformations du littoral français. L'île de Bouin (Vendée) à travers les âges d'après la géologie, la cartographie et l'histoire, Bulletin de géographie historique et descriptive, $\mathrm{n}^{\circ} 1-2$, p. 97-121. 
PÉRISSE S., 2011. Les campagnes saintongeaises à la fin du Moyen Âge (XV'-milieu XVI ${ }^{e}$ siècle), thèse université de La Rochelle, $579 \mathrm{p}$.

SArrazin J.-L., 1999. La paysannerie saunière des marais de la Baie à la fin du Moyen Âge : stratification et relations sociales, dans Antoine A. (dir.), Campagnes de l'Ouest. Stratigraphie et relations sociales dans l'histoire, Rennes, PUR, p. 185-200.

SArrazin J.-L., 2005. Le paysage salicole de l'île de Bouin à la fin du Moyen Âge, dans Chauvaud F., Peret J. (dir.), Terres marines. Études en hommage à Dominique Guillemet, Rennes, PUR, p. 57-67.
SARrAzin J.-L., 2008. La comptabilité de la seigneurie de Bouin en 1473-1474 d'après le compte de rachat de la baronnie de Rays, dans Cassard J.-C., Coativy Y., Gallice A., Le Page D. (dir.), Le prince, l'argent, les hommes au Moyen Âge. Mélanges offerts à Jean Kerhervé, Rennes, PUR, p. 283-298.

Verger F., 1968. Marais et wadden du littoral français, Bordeaux, Imprimerie Biscaye, 544 p.

Verger F., 2005. Marais et estuaires du littoral français, Paris, Belin, $335 \mathrm{p}$. 